\title{
The Predictability of Difficultness of Lower Third Molar Odontectomy
}

\author{
CIPRIAN IOAN ROI², EMILIA IANES ${ }^{1}$, PUSA NELA GAJ E², DIANA FLORINA NICA ${ }^{1}$, ANDREI ROI ${ }^{3}$, ALEXANDRA ROI ${ }^{4}$ \\ IVictor Babes University of Medicine and Pharmacy, Department of Anesthesiology and Oral Surgery, 2 Eftimie Murgu Sq.,300041, \\ Timisoara, Romania \\ ${ }^{2}$ Victor Babes University of Medicine and Pharmacy, Department of Microscopic Morphology/Histology, Angiogenesis Research \\ Center, 2 Eftimie Murgu Sq, 300041, Timisoara, Romania \\ ${ }^{3}$ Victor Babes University of Medicine and Pharmacy, 2 Eftimie Murgu Sq., 300041, Timisoara, Romania \\ ${ }^{4}$ Victor Babes University of Medicine and Pharmacy, Department of Oral Pathology, 2 Eftimie Murgu Sq., 300041, Timisoara, \\ Romania
}

The aim of this study was to establish an accurate algorithm for third molar odontectomy in order to help the surgeon to predict the duration and the difficultness of this surgical procedure. As material and methods, we used an algorithm that contains medical and radiographic data regarding lower wisdom tooth which was systematized in a preoperative questionnaire. In this study were included 27 patients with asymptomatic lower third molars during the period of 2015-2017. The prediction of the questionnaire was correlated with the difficulty and duration of the odontectomy. Results of our study showed that this algorithm can successfully predict the degree of difficulty of the lower wisdom tooth odontectomy and can guide the surgeon in the therapeutic decision. Moreover, this method is simple, easy and fast.

Keywords: third lower molar, odontectomy, predictability, algorithm, difficultness

After the decision for removal of the lower wisdom teeth is taken, we want to predict the difficulty and the duration of the odontectomy. In the literature we did not find a valid algorithm than can help the surgeon to predict the outcome of the intervention.

The reasons for the removal of the lower wisdom tooth can be prophylactic or curative. This procedure is made when the eruption predictability is unfavorable or if there are complications or there is no another method which can favor the eruption of the wisdom tooth. Third molar tooth has the higher variety and frequency of complications which can appear in the process of formation, development and eruption. Prophylactic odontectomy has the role of preventing local or general complications that can appear, but it should be taken into consideration the fact that the prophylactic removal continues to be a controversial issue and should be indicated depending on each case individually. The removal of impacted third molar is unarguably indicated when it is associated with pathologic complications that surpass the risks associated with the surgical procedure [1]. This complications can be inflammatory, septic, trophic, nervous or tumoral.

The main argument that supports the prophylactic odontectomy of the lower wisdom tooth was the lack of any role in the mouth. In one study, Wasiu [2] is claiming that in $18 \%$ to $50,7 \%$ of lower wisdom teeth odontectomies are not necessary. Prophylactic odontectomy should be based on the relationship between the benefits and the risks that may arise from this surgical procedure, the benefits outweigh the risks. The odontectomies are made to minimize the risk of developing cysts and tumors, decreasing in this way the risk of mandibular angle fracture. The incidence of the risks that are associated with the odontectomy of the third molars depend on the following factors: age, gender and health status (according to the American Society of Anesthesiologists), the bone density, the tooth position, the degree of impaction and also the experience of the surgeon who performs the intervention [3]. Some radiographic features suggest an increased risk of nerve damage associated with the removal of the mandibular third molar, and the study performed by L. Gu et. al [4] reveals the fact that there is a higher incidence of nerve involvement when the mandibular canal was lingually situated.

Following the NICE guidelines, they do not recommend routine, prophylactic extraction of pathology-free impacted mandibular third molar teeth. Their applicability has been questioned lately, although these are one of the mostrecent guidelines and recommendations. Their main inconvenient is the fact that they are based only on the expert opinion rather than evidence facts [5]. Following the urge to develop new updated guidelines, that should be based on an expert opinion, consultation and an amount of evidence proved facts, both the American Association of Oral and Maxillofacial Surgeons (AAOMFS), the Finnish Medical Society Duodecim and Finnish Dental Society Apollonia working group have recently published guidance on the removal of mandibular third molar teeth [6].

The impaction rate is higher for the third molars than for any other tooth and especially the eruption space for the third mandibular molar is perturbed during the function eruption phase. The statistics prove the fact that about $40 \%$ of the lower third molars become partially or fully impacted [7].

A number of authors have conducted numerous studies trying to find different correlations and patterns that could predict if wisdom tooth will erupt or not, if it has place in the mandibular arch or the angulation of the third molar position will be vertical, perpendicular to the occlusal plane, in mesial or distal position.

Richardson [8] tried to determine the relationship between the angulation of lower third molar and various dimensions of the jaws and teeth.Measurements they performed on radiographs were the angle between the occlusal surface of lower third molar, mandibular plane passing through $\mathrm{Gn}$ and Go- noted $\mathrm{Y} 1$ and the angle between the occlusal surface of lower third molar and mandibular occlusal plane, noted Y2. 


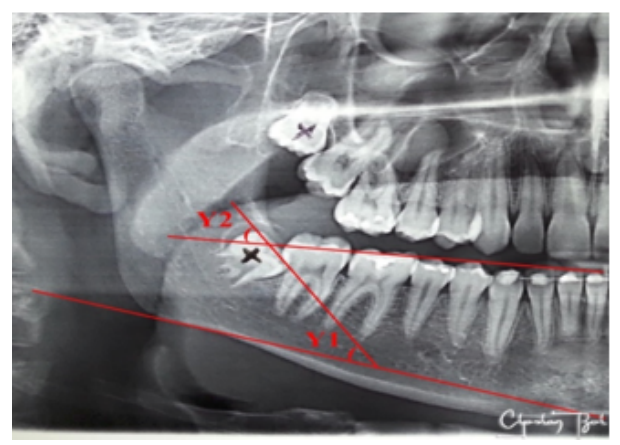

Ganss [9], outlined the importance of space correlation between dimensions of retro molar space and the chances of eruption of inferior third molars.

\section{Experimental part}

Materials and methods

In this study were included 27 patients admitted in Oral and Maxillofacial Surgery Timisoara Hospital for lower third molar odontectomy in 2015, 2016 and 2017. The inclusion criteria for this study, were: both sexes, age ranging from 14 to 70 years, wisdom tooth on the right and/or the left

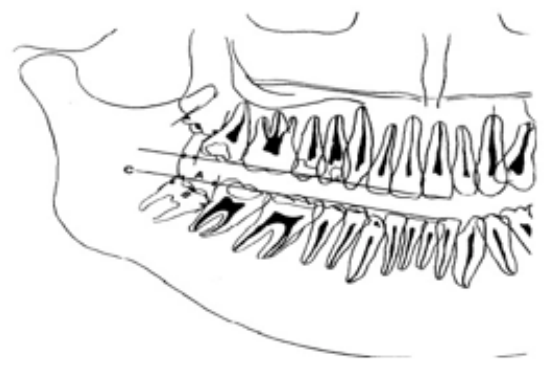

Fig.2. Ganss space correlations side of the mandible), lower wisdom teeth will be subject for odontectomies, lower wisdom teeth partially or fully impacted, lower third molars in different stages of development, wisdom teeth on which surgery was not already applied, wisdom teeth that do not show acute infectious complications.

Each patient was subjected to an orthopantomographic examination, scale 1:1, using the same incidence. The following questionnaire was applied for each patientbefore the surgical procedure:

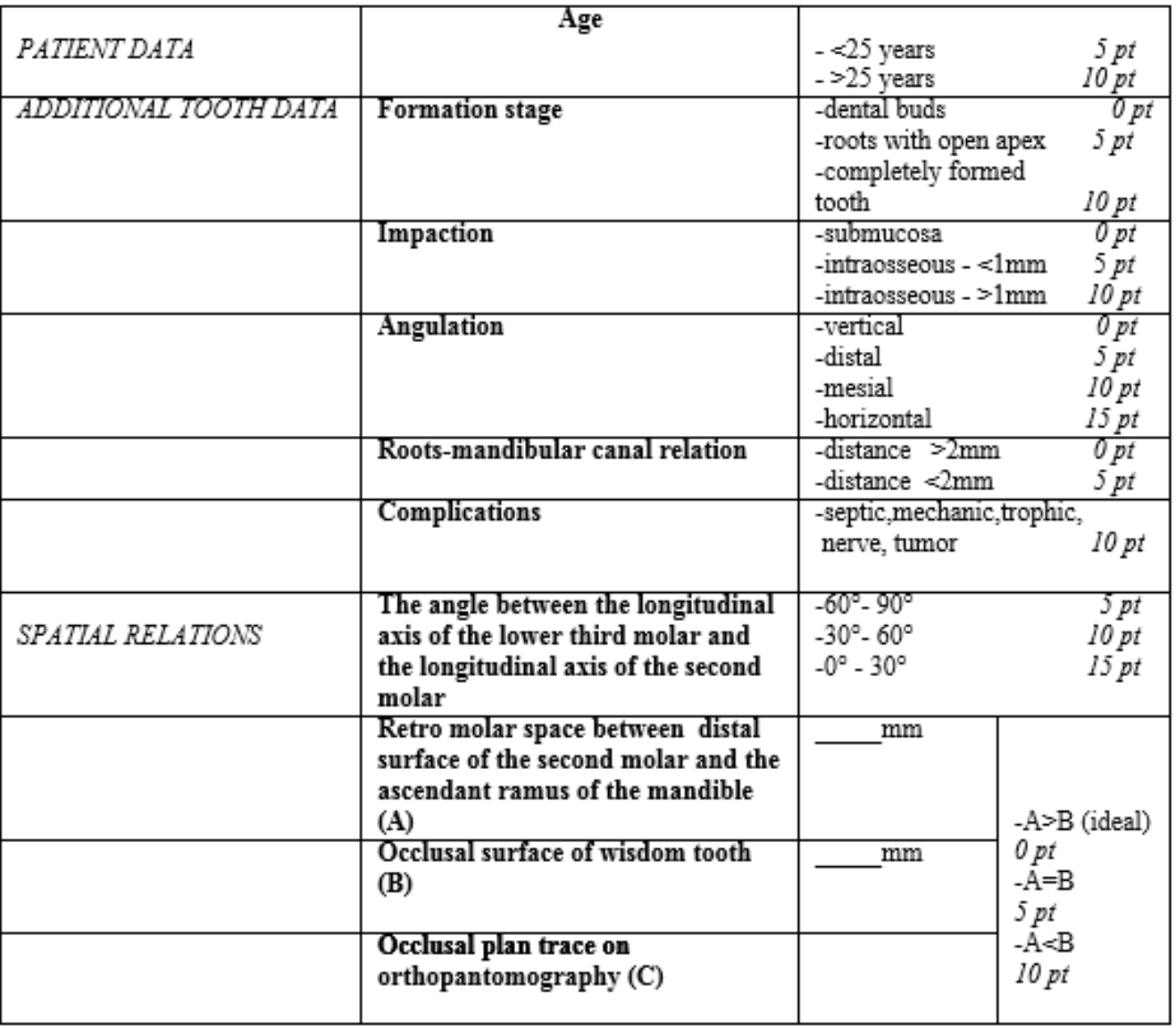

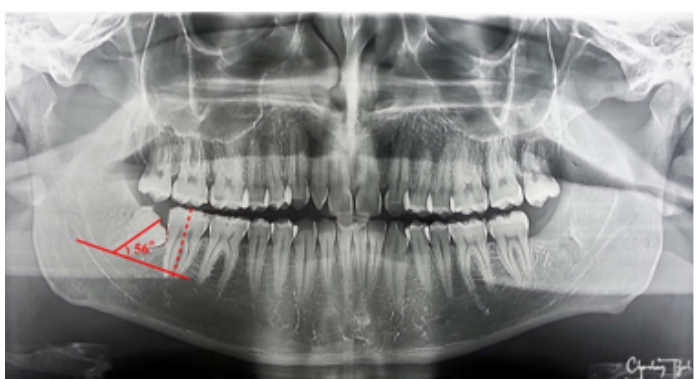

Fig.3. The angle between the longitudinal axis of the lower third molar and the longitudinal axis of the second molar

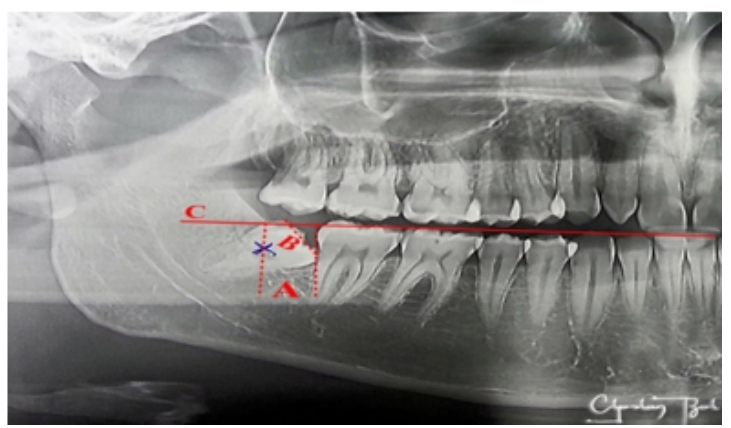

Fig.4. Spatial relations of the right third lower tooth 
To the end of the questionnaire, the points will be summed up, and the resultcan predict the difficulty of the odontectomy:

-0-10 points: LOW LEVEL OF DIFFICULTY;

-11-50 points: MEDIUM LEVEL OF DIFFICULTY;

-51-85 points: HIGH LEVEL OF DIFFICULTY.

\section{Results and discussions}

In this study were included 27 patients, 11 men and 16 women, which means $40.74 \%$ men and $59.26 \%$ women. Patients under 25 years- 22 patients- $81.48 \%$, only 2 patients$18.52 \%$ were over 25 years. The most involved tooth was inferior right third molar- 18 molars were located on right mandibular arch-66.66\% and on left mandibular arch 9 molars $-33.34 \%$. Regarding the tooth formation, 10 patients $-37.03 \%$ were in dental buds formation stage, 2 patients $7.40 \%$ roots with open apex, 15 patients- $55.55 \%$ has completely formed tooth. The molar impaction was: submucosa impaction, 15 patients- $55.55 \%$, intraosseous $<1 \mathrm{~mm} 11$ patients- $40.74 \%$, intraosseous $>1 \mathrm{~mm} 1$ patient $-3.70 \%$. Regarding the angulation of the wisdom teeth, the mesial one was the most frequent, 19 patients $-70.37 \%$; the other impaction forms were vertical angulation 3 patients $-11.11 \%$, distal angulation- $0 \%$, horizontal 5 patients $-18.51 \%$. As other studies reveled, the majority of the wisdom tooth were located near the mandibular canal: distance $>2 \mathrm{~mm} 2$ patients $-7.40 \%$, distance $<2 \mathrm{~mm} 25$ patients $-92.59 \%$ and has complications in history 23 patients $-85.18 \%$. How about spatial relations of the lower wisdom teeth, the angle between the longitudinal axis of the lower third molar and the longitudinal axis of the second molar was between 60 and $90^{\circ}$ in the majority of the cases 15 patients $-55.55 \%$, between 0 and $30^{\circ} 4$ patients $-14.81 \%$, between 30 and $60^{\circ} 8$ patients $-29.62 \%$ and there was no enough space for the eruption of the third molar, this fact justifies the therapeutic odontectomy, because $A>B 1$ patient $-3.70 \%, A=B 1$ patient $-3.70 \%, A<B 25$ patients $92.59 \%$. Correlating the questionnaire with the odontectomy, the difficulty level of the odontectomy were in majority of the cases medium or high, with a single one case difference: low level of difficulty $0 \%$, medium level of difficulty 14 patients $-51.85 \%$, high level of difficulty 13 patients $-48.15 \%$.

Previous studies tried to to predict the probability of third molar eruption using dissected skulls and lateral cephalometric radiographs [7]. The results of the studies have proved the fact that from the panoramic radiographs we can obtain pertinent measurements as that of lateral skull radiographs, with the advantage that the left and right sides can be measured separately in panoramic radiographs. The evolution that the digital technology offers allows image adjustments that can successfully contribute to an accurate clinical perspective [10].

Carvalho and Vasconcelos [11] made 473 odontectomies of lower wisdom teeth and concluded that the number of roots, tooth position, periodontal space and second molar relation were significant predictors of the odontectomy difficulty. In another study, Akadiri and Obiechina [12] demonstrated that the third molar depth angulation and root morphology are the most consistent determinants of extraction difficulty.

Trying to minimize the number of complications and accidents during and after the odontectomies, several classifications have been developed. The most used are Winter [13] and Pell and Gregory [14] classifications which analyzes the angulations and the positions of the lower third molars based on the relation between the dental longitudinal axis, occlusal plane and ascending mandibular ramus [15]. However some authors claim that these scales have little value for predicting the degree of extraction difficulty [16], mainly because these systems of classification introduce error of interpretation by the observer [17].

In our study, regarding the age, the patients were under 25 years and more than 25 years, because after 25 years, the elasticity of jaws is decreasing by higher levels of inorganic matrix. So, mandibular bone is more firm and the difficulty of odontectomy is increased.

Regarding the additional tooth data field from our questionnaire, we recorded some extra datas about the tooth. The first parameter analyzed was the formation stage. When the third molar is in dental buds stage and the patient is a teenager, the alveolar bone is elastic, the tooth does not have a strong bone implantation, so, the difficulty of odontectomy is on a lower scale. On the other side, when the third molar is developed, bone implantation is stronger and the tooth has found his position in alveolar bone, so, the difficulty of odontectomy is higher. The impaction was the second parameter analyzed. Submucosa impactation is the easiest situation regarding the difficulty of the odontectomy because only a flap to have access to the tooth is necessary. When the impaction is intraosseous, the difficulty of the odontectomy is higher, because osteotomy is needed. The third parameter included was the angulation of the third inferior molar. The most favorable is vertical position of lower wisdom tooth, which has the distal face free of mandibular bone. The worst situation is the horizontal position, when is necessary crown and roots dissection to facilitate the extraction of the tooth. Another parameter was the relation between the roots of the third molar and mandibular canal. When wisdom tooth roots are near the mandibular canal, the difficulty of the odontectomy rises and the risk of sectioning or elongating the inferior alveolar nerve is high. The last additional parameters of the tooth that were recorded, were the septic, mechanic, trophic, nerve or tumor complications of the wisdom tooth.

Regarding the spatial relations field from our questionnaire, the angle between the longitudinal axis of the lower third molar and the longitudinal axis of the second molarwas recorded. The angle between the longitudinal axis of the lower third molar and the longitudinal axis of the second molar can predict the chances of eruption: if the angle is between 0 and $30^{\circ}$ - SEVERE prognosis, with a high risk for the wisdom tooth to remain inmpacted; if the angle is between 30 and $60^{\circ}$ - MEDIUM prognosis; if the angle is between 60 and $90^{\circ}$ - FAVORABLE prognosis, with chances of spontaneous eruption.

Other measurements were made on the radiography: retro molar space between distal surface of the second molar and the ascendant ramus of the mandible $(A)$ :

$\mathrm{mm}$, occlusal surface of wisdom tooth $(B)$ : $\mathrm{mm}$, occlusal plane trace on orthopantomography ( $\bar{C})$. If $A>B$, the third molar has space for eruption on the dental arch and the doctor can wait with the odontectomy. In the other two situations, $A<B, A=B$, the right decision is the odontectomy. When the value of $A$ is low, the difficulty of the odontectomy is higher.

To the end of the questionnaire, the sum of the points is made, and the result can predict the difficulty of the odontectomy: 0-10 points: LOW LEVEL OF DIFFICULTY; 11-50 points: MEDIUM LEVEL OF DIFFICULTY; 51-85 points: HIGH LEVEL OF DIFFICULTY.

It could be speculated the fact that asymptomatic mandibular third molars positioned either mesioangulated or horizontally could be removed based on the panoramic 
radiography [18] and a correct analysis. An increased risk of pathology observed on the radiography and involving the mandibular third molars positioned in either of these angulations should provide enough evidence for the indication of tooth removal.

\section{Conclusions}

On the basis of the present results, our algorithm can predict the difficulty of the odontectomy of the lower third molar. Our method is valuable, cost effective, fast and easy to apply. It can guide the surgeon in the therapeutic decision, avoiding the accidents and unpleasant surprises that may occur.

\section{References}

1. SINGH, K., KUMAR, S., SINGH, S., MISHRA, V., SHARMA, P.K., SINGH

D. Impacted mandibular third molar: Comparison of coronectomy with odontectomy. Indian J Dent Res, 29,2018, p.605.

2. ADEYEMO,W.L. Do pathologies associated with impacted lower third molars justify prophylactic removal? A critical review of the literature. Oral Surgery, Oral Medicine, Oral Pathology, Oral Radiology, Endodontics, 102, 2006, p.448

3. MANOR, Y., ABIR , R., MANOR, A., KAFFE, I. Are different imaging methods affecting the treatment decision of extractions of mandibular third molars?. Dentomaxillofacial Radiology, 46, 2017, p. 1-6.

4. GU, L., ZHU, C., CHEN, K. ET AL. Anatomic study of the position of the mandibular canal and corresponding mandibular third molar on cone-beam computed tomography images, Surg Radiol Anat, 40, nr. 6,2018, p.609.

5. ALI, A. S., BENTON, J. A., YATES, J. M. Risk of inferior alveolar nerve injury with coronectomy vs surgical extraction of mandibular third molars-A comparison of two techniques and review of the literature. Journal of Oral Rehabilitation, 45(3), 2017, p. 250.

6 .*** Guidelines on the management of lower third molars. Finland: Kaypa Hoito/Current care guidelines. 2014. http://ww w.kaypahoito.fi/ web/english/guidelines/guideline?id $=c c g 00003 \&$ suositusid $=c c s 00056$. 7. KAUR, R., KUMAR, A.C., GARG, R., SHARMA, S., RASTOGI, T., GUPTA, V.V. Early prediction of mandibular third molar eruption/impaction using linear and angular measurements on digital panoramic radiography: A radiographic study. Indian J Dent, 7. nr.2, 2016, p.66.
8. RICHARDSON,M.E. The early developmental position of the lower third molar relative to certain jaw dimensions, The Angle Orthodontist, 40,1970, p. 226-230;

9. GANSS,C., HOCHBAN,W., KIELBASSA, A.M. Prognosis of Third Molar Eruption, Oral surgery, medicine and pathology J ournal,76, nr.6,1993, pg. 688.

10. UTHMAN, A.T. Retromolar space analysis in relation to selected linear and angular measurements for an Iraqi sample. Oral Surg Oral Med Oral Pathol Oral Radiol Endod, 104,2007,p.76-82.

11. CARVALHO, R.W., DO EGITO VASCONCELOS, B.C. Assessment of factors associated with surgical difficulty during removal of impacted lower third molars. International J ournal of Oral and Maxillofacial Surgery, 69, nr. 11, 2011, p. 2714.

12. AKADIRI, O.A., OBIECHINA, A.E. Assessment of difficulty in third molar surgery--a systematic review. International J ournal of Oral and Maxillofacial Surgery, 67, nr.4, 2009, p.771.

13. WINTER, G.B. Principles of exodontia as applied to the impacted mandibular third molar; a complete treatise on the operative technic with clinical diagnoses and radiographic interpretations, St Louis: American Medical Book, 1926. p. 241.

14. PELL, G.J., GREGORY, B.T. Impacted mandibular third molars: classification and modified techniques for removal. Dent Digest, 39, 1933, p.330.

15. JUODZBALYS, G., DAUGELA, P. Mandibular Third Molar Impaction: Review of Literature and a Proposal of a Classification, Journal of Oral and Maxillofacial Research, 4, nr. 2,e1, 2013.

16. GARCIA, A.G., SAMPEDRO, F.G., REY, J.G., VILA, P.G., MARTIN, M.S. PellGregory classification is unreliable as a predictor of difficulty in extracting impacted lower third molars. British J ournal of Oral and Maxillofacial Surgery, 38, nr.6, 2000.

17.ALMENDROS, M. N., BERINI, A.L., GAY, E.C. Evaluation of intraexaminer and interexaminer agreement on classifying lower third molars according to the systems of Pell and Gregory and of Winter. International J ournal of Oral and Maxillofacial Surgery, 66, nr. 5, 2008. 18. MATZEN, L.H., SCHROPP, L., SPIN-NETO, R., WENZEL, A. Radiographic signs of pathology determining removal of an impacted mandibular third molar assessed in a panoramic image or CBCT. Dentomaxillofacial Radiology, 46, 2017, p. 1-8.

Manuscript received: 3.10 .2018 